\title{
Pharmaceutical Applications of Circular Dichroism for
}

\section{Nanomaterial's}

\section{Manish Kumar* and Abhishek Jha}

Department of Pharmaceutical Engineering and Technology, Indian Institute of Technology (Banaras Hindu University), Varanasi- 221005, India

*Corresponding author: Manish Kumar, Department of Pharmaceutical Engineering and Technology, Indian Institute of Technology (Banaras Hindu University), Varanasi-221005; Email: manishkumar.rs.phe19@itbhu.ac.in

\section{Abstract}

Circular dichroism is a powerful, fast, non-destructive analytical technique for small molecules and big molecules such as proteins, peptides etc. Here, a basic review on principle and advantages of CD is given followed by application in various studies such as molecular interaction among chiral molecules, drug bio-target interaction, nanoparticle protein interaction, biopolymers, chiral molecule grafted nanocarriers, chiral molecule loaded Nano carriers and chiral nanoparticles. In conclusion, circular dichroism is a powerful tool that can be used for conducting various interactions studies such as protein-receptor interaction, ligand or drug-protein interaction, confirming the siRNA/ siDNA delivery, determining any change, chemical modification or mutation in target, if occurred.

Keywords: Circular Dichroism; Nanomaterial's; Mutations; Nano carriers

\section{Introduction}

Circular dichroism (CD) spectroscopy is widely applicable in the pharmaceutical industry to study the effects of different processing conditions (such as composition of formulation, storage conditions, delivery system, etc.) on protein conformation and stability [1]. CD is the method of choice for elucidating and examining the structural actions of proteins, lipids, nucleic acids, carbohydrates, chiral molecules, and so on, even when present in solution [2]. CD is the discrepancy between a chiral moiety's right and left polarized light absorption and due to absorption in the UV-Visible and IR regions, electronic and vibrational CDs are generated respectively [2]. CD instrument divides the plane polarized light into right and left circularly polarized lights by passing it through a modulator, composed of a piezoelectric-quartz crystal and a thin plate. Structural change induced by alternating electrical field in quartz cell results in the transmission of circularly polarized light by plates as LCP and RCP lights, later detected by photomultipliers. Here, the chiral sample absorbs any one light to a greater extent resulting in elliptical lights polarization, traced as an ellipse. A CD spectrum is observed as a function of wavelength, indicating variations in chiral molecule absorption of LCP and RCP beams [3].

\section{Advantages and Limitations of CD}

- The characterisation can be carried out in an aqueous state that is more real to the understanding of proteins and their biological functions as the proteins actually work in solution. In contrast characterization by NMR 
and X-ray crystallography requires crystal molecular forms [3].

- The spectrum of single protein UV far CD over the range of 190-240 nm may take less than 3 minutes; while titration tests and temperature based measuring (e.g. ligand or DNA proteins) usually take 1-2 hours.

- Studies can be performed to elucidate structure under wide aqueous conditions such as varying concentrations, different $\mathrm{pH}$ and temperature, ionic, detergent condition etc $[4,5]$.

- Do not require pre-treatment as required in NMR and X-ray crystallography for glycoproteins or membrane proteins as CD bands for protein components are not disturbed by carbohydrate and residual lipids [3].

- Provide low resolution-structural information for small molecules and biopolymers, a major limitation of this method compared to NMR and X-ray crystallography [3].

- Used for protein and peptide analysis, conformational behaviour and protein ligand/drug interaction $[6,4]$.

\section{Applications}

The stereoisomers properties in modern medicines are necessary according to the regulations of Food and Drug Administration (FDA). Far UV and Near-UV CD spectrum allow the correct folding and conformations to be verified, which can help to determine structural modifications during formulation, processing, release, administration and monitor protein sample impurities.

\section{Chiral Molecule and CD}

$\mathrm{CD}$ is an excellent technique to determine and differentiate optically active or chiral drug or molecules from other non-chiral or chiral molecules. Example quantification of ampicillin from a mixture containing cloxacillin [7].

\section{Bio-Polymer and CD}

CD can be used to study proteins secondary and tertiary structures in a solution as it can detect $\alpha$ helical sheets, $\beta$ sheet and conformations. Though a comparison study for two or more spectra is required to confirm stability of protein conformation. Few approaches such as Overlay CD spectra method, comparison of mean residue ellipticity (MRE) of specific CD spectra and deconvolution of the far-UV CD spectra have been used to compare CD spectra and assess overall similarity, but have limitations. Method of overlay CD spectra may lead to false positive or negative determinations. MRE is used only to compares the spectra at definite wavelengths instead of over the entire spectral range. For example, a broad signal at 250$280 \mathrm{~nm}$ for disulfide conformation and signal at 250-265 $\mathrm{nm}$ for phenylalanine can superimposed each other, difficult to differentiate. Though, deconvolution of the farUV CD spectrum of a protein elucidated secondary structural components ( $\alpha$ helix, $\beta$ sheet, $\beta$ turn, unordered conformation, contributions by aromatic amino acids at 195-240 nm) as well as determined the percentage of each component. Later, comparative study could be done to determine percentage component and structural change [1].

\section{Nanoparticles-Protein Interaction and CD}

A study was conducted to insight the binding between bovine serum albumin (BSA) and gold nanoparticles (GNPs) or silver nanoparticles (SNPs) for targeted drug delivery. The study was done to observe any possible interaction between gold or silica nanoparticles with glycan and thus study protein-ligand and protein-protein interactions. Here, CD Spectral measurements were performed from 190 to $250 \mathrm{~nm}$ (far-UV) for BSA, BSA-GNP and BSA-SNP incubated with glycan (D-mannose (MAN), L-fructose (FUC), D-galactose (GAL), $\mathrm{N}$-acetyl glucosamine (NAG)). Mean Residue Ellipticity (MRE) in degree $\mathrm{cm}^{2}$ $\mathrm{dmol}^{-1}$ was determined by using formula

$$
M R E_{208 \mathrm{~nm}}=\frac{\theta_{208 \mathrm{~nm}}}{C_{p} \times n \times l \times 10}
$$

Where $\theta_{208} \mathrm{~nm}=\mathrm{CD}$ in $\mathrm{mdeg}, \mathrm{C}_{\mathrm{p}}=$ concentration in $\mathrm{mg} / \mathrm{ml}$, $\mathrm{n}=$ number of amino acids and $\mathrm{l}=$ path length of the cell. Ahelix content was also measure by using following equation

$$
\alpha \operatorname{helix}(\%)=\left[\frac{-M R E_{208 n m}-4000}{33000-4000}\right] 100
$$

$\mathrm{MRE}_{208 \mathrm{~nm}}=$ observed MRE value at 208nm, 4,000 =MRE of the $\beta$ form and random coil conformation at $208 \mathrm{~nm}$ and $33,000=$ MRE value of a pure helix at $208 \mathrm{~nm}$. Results indicated that GNP and SNP not contribute to the CD signal where two negative peaks observed at $208 \mathrm{~nm}$ and $222 \mathrm{~nm}$ of $\alpha$-helix of BSA and a strong positive peak at $\sim 190 \mathrm{~nm}$ was observed which belongs to $n \rightarrow \pi^{*}$ transfer and $\pi \rightarrow \pi^{*}$ transition respectively. The $\alpha$-helix content of BSA was to normal value (65.47\%) thus BSA secondary structure was intact on adsorption to GNP or SNP, showing insignificant changes in the peaks at 208 and 222 nm. Finally, chiral nature of NANA, MAN, GAL, and FUC was interfered with CD spectra of BSA, BSA-GNP, and BSA-SNP. For NAG, a loss in signals at $208 \mathrm{~nm}$ and $222 \mathrm{~nm}$ 
was observed which indicated the disruption in helical structure and loss of rigid tertiary structure due to the asymmetrical environment around aromatic chromophore. Thus, the BSA-GNP and BSA-SNP maintained the secondary structure stability and had insignificant conformational change [8].

\section{Drug-Biotarget Interaction and CD}

Similarly, CD was used to conduct interaction study for a drug with protein. For example procainamide hydrochloride (PAH) with human serum albumin (HSA) [9]. The two negative bands at 208 and $220 \mathrm{~nm}$ characteristic to $\alpha$ helix structure of HSA were observed on CD spectrum. The $\alpha$-helicity in secondary structure of HSA was decreased from $81.09 \%$ to $62.43 \%$ in PAH and HSA complex, indicating of the loss of $\alpha$-helicity upon interaction. Further, no significant shift in peaks of HSAs CD spectra in the presence and absence of PAH was observed, thereby indicating maintenance of integrity of structure of HSA even after binding. Binding of drug to target with molecular mechanism can also be verified by use of CD. Sunitinib can results in shifting of CD bands of DNA (two plateau observed) and appearance of an induced signal at about $359 \mathrm{~nm}$, confirming of binding sunatinib to DNA. The mechanism for binding involve unwinding of superhelical structure of DNA due to interaction of drug with groove of DNA followed by electrostatic binding of drug side chain to phosphate group of DNA via protonate nitrogen [10].

\section{Chiral Molecule Grafted Nanocarriers and CD}

Chiral molecule grafting on the surface of nanocarriers can provide improved drug delivery behaviour and can have specific biological recognition. CD Analysis can be done to confirm the attachment of chiral molecules to the substrate. Chiral molecule can provide enantioselective cellular uptake in cancerous cells. For example cellular uptake for chiral quantum dots (QD) based on L Cysteine was twice of D Cystein QDs. Here, a pronounced opposite optical dichroism signal in the spectral region of the $\mathrm{D}$ and L Cys QDs was observed which confirm the formation of the enantiomeric D and L chiral QDs. Thus in order to confirm the preparation of chiral systems, circular dichroism (CD) study could be conducted [11]. Similarly, Yingyu G, et al. prepared chiral molecule grafted mesoporous silica nanoparticles (MSNs). Levorotatory MSNs (erected peak at $218 \mathrm{~nm}$ ) and dextrorotatory MSNs (while reversal peak at $218 \mathrm{~nm}$ ) verified by CD spectra, provided the oral bioavailability of nimesulide upto $698.45 \%$ and $887.03 \%$ respectively. The superior chiral response performance of D-MSNs presented stronger anti-inflammatory pharmacodynamics performance [12].

\section{Chiral Molecule Loaded Nano carriers and CD}

CD techniques can help to confirm any possible change in conformational structure and integrity of protein which can occur while loading inside the nanocarriers. For desired action, loaded protein responsible for a specific activity should not undergo any change in secondary structure or its conformation which can be confirmed by circular dichroism. For example an injectable protein drug delivery system was prepared to load model protein (enzyme $\alpha$ amylase) where no change in confirmation was observed before and after release [13]. Similarly, Erel $\mathrm{G}$, et al. used CD analysis to verify no change in conformation stability for insulin while preparation of insulin encapsulated chitosan nanoparticles [14].

\section{Chiral Nanoparticles and CD}

Recently, chiral nanoparticles have gained wide attention and attracted many researchers due to its potential applications. Lin J, et al. prepared chiral ZnO nanoparticles using l-cysteine as ligand and used these nanoparticles as probe for detection of dopamine in urine samples or bioanalysis [15]. CD spectra for free cysteine are at 209nm, though peak observed for ZnO@Cys is $246 \mathrm{~nm}$ which may be due to strong interaction between $\mathrm{ZnO}$ and cysteine. ZnO@Cys found to have additional CD peaks $(324 \mathrm{~nm}, 374 \mathrm{~nm}$ ) compared to $\mathrm{ZnO}$ and cysteine. Hence, cysteine adsorbed on NPs surfaces not only preserved own chirality but also induced the chirality to $\mathrm{ZnO}$ nanoparticles providing new potential applications as biosensors.

CD provides an insight to conformational changes which can occur in protein used for preparation of nanoparticle. For example Casein, a milk protein form micelles in aqueous solution, can be used to prepare casein nanoparticles loaded with an anticancer drug. CD showed that excess $\mathrm{Ca} 2+$ added, rearranged the protein chains leading to more 'packing' and conversion of nanomicelles to dense nanoparticles. CD of Sodium caseinate showed two negative bands at $208 \mathrm{~nm} \& 222 \mathrm{~nm}$ and a positive band around $193 \mathrm{~nm}$ for $\alpha$ helices. Upon particle formation (addition of Ca2+ ions), magnitude of bands decreased with hardly any ellipticity beyond 210 $\mathrm{nm}$ due to complete loss of $\alpha$-helices. Possibly Ca2+ ions interacted with phosphoserine residues of casein acting as a bridge leading to rearrangement of the casein molecules (soluble in water as well as micellar) to 
maximize the interactions between added $\mathrm{Ca}^{2+}$ ions and phosphoserine residues present [16].

CD can be used to understand how and where the drug locates itself inside polymeric nanocarriers. Wankar J, et al. used CD to explain that the drug (ethionamide) interact and locate itself in polymeric betacyclodextrin (pbCyD) cavity and microdomains or confined spaces present inside the cross linked polymer to give drug loaded nanoparticle [17]. Here, drug induced a CD peak due to experienced chiral environment by polymeric cyclodextrin (negative signal at $280 \mathrm{~nm}$, positive band at $320 \mathrm{~nm}$ and intense shoulder at $380 \mathrm{~nm}$ ). Moreover different concentration of polymer found to give similar spectra but relative intensities of bands varied for two complexes, reflecting different environments for complexation. Liu J, et al. also demonstrated that no change in aptamers G-quadruplex structure was observed on addition of dsDNA and Dox by employing CD analytical technique. The aptamer used to prepare dsDNA and Dox loaded nanoparticle found to maintain G-quadruplex parallel strand conformation (positive peak at $262 \mathrm{~nm}$ and a negative peak at $238 \mathrm{~nm}$ ) which is crucial for action [18].

\section{Conclusion}

Circular dichroism is a powerful, fast, non-destructive analytical technique for studying structure and ligand interaction of small molecules. Here, initially a basic review to principle and advantages of technique was discussed followed by a systematic literature review on pharmaceutical aspects which included the use of CD analytical technique for various study such as chiral molecule interaction study, biopolymer study, understand drug bio-target interaction and nanoparticle protein interaction, confirm preparation of chiral molecule grafted Nano carriers, chiral molecule loaded Nano carriers and chiral molecules nanoparticle and so on. In conclusion, circular dichroism can prove to be a powerful and versatile tool for conducting study of chiral nanomaterial's and various interactions such as protein receptor interaction, ligand-protein interaction, change in target protein, chemical modification in biomolecules, study mutation etc.

\section{References}

1. Li CH, Nguyen X, Narhi L, Chemmalil L, Towers E, et al. (2011) Applications of circular dichroism (CD) for structural analysis of proteins: qualification of near-

Manish Kumar and Abhishek Jha. Pharmaceutical Applications of Circular Dichroism for Nanomaterial's. Adv Clin Toxicol 2019, 4(4): 000173. and far-UV CD for protein higher order structural analysis. Journal of Pharmaceutical Sciences 100(11): 4642-4654.

2. Siligardi G, Hussain R (2017) Circular Dichroism, Applications. In: Lindon JC, et al. [Eds.], Encyclopedia of Spectroscopy and Spectrometry (Third Edition), pp: 293-298.

3. Yao H, Wynendaele E, Xu X, Kosgei A, De Spiegeleer B (2018) Circular dichroism in functional quality evaluation of medicines. J Pharm Biomed Anal 147: 50-64.

4. Siligardi G, Hussain R, Patching SG, Phillips-Jones MK (2014) Ligand- and drug-binding studies of membrane proteins revealed through circular dichroism spectroscopy. Biochim Biophys Acta BBA Biomembr 1838(1Pt A): 34-42.

5. Miles AJ, Wallace BA (2015) Chapter 6-Circular Dichroism Spectroscopy for Protein Characterization: Biopharmaceutical Applications. In: Houde DJ, et al. [Eds.], Biophysical Characterization of Proteins in Developing Biopharmaceuticals, pp: 109-137.

6. Greenfield NJ (1999) Applications of circular dichroism in protein and peptide analysis. TrAC Trends Anal Chem 18(4): 236-244.

7. Rahman N, Khan S (2016) Circular dichroism spectroscopy: An efficient approach for the quantitation of ampicillin in presence of cloxacillin. Spectrochim Acta A Mol Biomol Spectrosc 160: 26-33.

8. Kumari N, Mathe VL, Dongre PM (2019) Albumin nanoparticles conjugates binding with glycan - A strategic approach for targeted drug delivery. Int J Biol Macromol 126: 74-90.

9. Byadagi K, Meti M, Nandibewoor S, Chimatadar S (2017) Investigation of binding behaviour of procainamide hydrochloride with human serum albumin using synchronous, 3D fluorescence and circular dichroism. J Pharm Anal 7(2): 103-109.

10. Kiss E, Mirzahosseini A, Hubert A, Ambrus A, Orfi L, et al. (2018) DNA binding of sunitinib: Spectroscopic evidence via circular dichroism and nuclear magnetic resonance. J Pharm Biomed Anal 150: 355-361.

11. Martynenko IV, Kuznetsova VA, Litvinov IK, Orlova AO, Maslov VG, et al. (216) Enantioselective cellular uptake of chiral semiconductor nanocrystals. Nanotechnology 27(7). 
12. Guo Y, Wu L, Gou K, Wang Y, Hu B, et al. (2019) Functional mesoporous silica nanoparticles for delivering nimesulide with chiral recognition performance. Microporous Mesoporous Mater.

13. Alcala-Alcala S, Benitez-Cardoza CG, Lima-Munoz EJ, Pinon-Segundo E, Quintanar-Guerrero D (2015) Evaluation of a combined drug-delivery system for proteins assembled with polymeric nanoparticles and porous microspheres; characterization and protein integrity studies. Int J Pharm 489(1-2): 139-147.

14. Erel G, Kotmakcı M, Akbaba H, Sozer Karadagli S, Kantarci AG (2016) Nanoencapsulated chitosan nanoparticles in emulsion-based oral delivery system: in vitro and in vivo evaluation of insulin loaded formulation. J Drug Deliv Sci Technol 36: 161167.
15. Lin J, Huang B, Dai Y, Wei J, Chen Y (2018) Chiral ZnO nanoparticles for detection of dopamine. Mater Sci Eng C 93: 739-45.

16. Gandhi S, Roy I (2019) Doxorubicin-loaded casein nanoparticles for drug delivery: Preparation, characterization and in vitro evaluation. Int J Biol Macromol 121: 6-12.

17. Wankar J, Salzano G, Pancani E, Benkovics G, Malanga $M$, et al. (2017) Efficient loading of ethionamide in cyclodextrin-based carriers offers enhanced solubility and inhibition of drug crystallization. Int J Pharm 531(2): 568-576.

18. Liu J, Wei T, Zhao J, Huang Y, Deng H, et al. (2016) Multifunctional aptamer-based nanoparticles for targeted drug delivery to circumvent cancer resistance. Biomaterials 91: 44-56. 